Research Paper

\title{
Profiles of Immune Infiltration in Bladder Cancer and its Clinical Significance: an Integrative Genomic Analysis
}

\author{
Zonglong $\mathrm{Wu}^{1,2^{*}}$, Kejia Zhu ${ }^{1,2^{*}}$, Qinggang Liu1,2, Yaxiao Liu ${ }^{1,2}$, Lipeng Chen ${ }^{1,2}$, Jianfeng Cui ${ }^{1,2}$, Hongda \\ Guo $^{1,2}$, Nan Zhou ${ }^{1,2}$, Yaofeng Zhu ${ }^{1,2}$, Yan Li1 ${ }^{1,2}$, Benkang Shi ${ }^{1,2}{ }^{\bowtie}$ \\ 1. Department of Urology, Qilu Hospital of Shandong University, Jinan, P.R. China. \\ 2. Key Laboratory of Urinary Precision Diagnosis and Treatment in Universities of Shandong, Jinan, P.R. China. \\ *These authors contributed equally to this work. \\ $\triangle$ Corresponding authors: Yan Li, Department of Urology, Qilu Hospital of Shandong University, 107 Wenhuaxi Road, Jinan, 250012, China, E-mail: \\ yanli@sdu.edu.cn. Benkang Shi, Department of Urology, Qilu Hospital of Shandong University, 107 Wenhuaxi Road, Jinan, 250012, China, E-mail: \\ bkang68@sdu.edu.cn. \\ (c) The author(s). This is an open access article distributed under the terms of the Creative Commons Attribution License (https://creativecommons.org/licenses/by/4.0/). \\ See http://ivyspring.com/terms for full terms and conditions.
}

Received: 2019.11.16; Accepted: 2020.02.20; Published: 2020.03.05

\begin{abstract}
Tumor-infiltrating immune cells are closely related to the prognosis of bladder cancer. Analysis of tumor infiltrating immune cells is usually based on immunohistochemical analysis. Since many immune cell marker proteins are not specific for different immune cells, which may induce misleading or incomplete. CIBERSORT is an algorithm to estimate specific cell types in a mixed cell population using gene expression data. In this study, the CIBERSORT algorithm was used to identify the immune cell infiltration signatures. The gene expression profiles, mutation data, and clinical data were collected from The Cancer Genome Atlas (TCGA) database. Unsupervised consensus clustering was used to acquire the immune cell infiltration subtypes of bladder cancer based on the fractions of 22 immune cell types. Four immune cell clusters with different immune infiltrate and mutation characteristics were identified. In addition, this stratification has a prognostic relevance, with cluster 2 having the best outcome, cluster 1 the worst. These clusters showed distinct mRNA expression patterns. The characteristic genes in subtype cluster 1 were mainly involved in cell division, those in subtype cluster 2 were mainly related in antigen processing and presentation, those in subtype cluster 3 were mainly involved in epidermal cell differentiation, and those in subtype cluster 4 were mainly related in the humoral immune response. These differences may affect the development of the bladder cancer, the sensitivity to treatment as well as the prognosis. Through further validation, this study may contribute to the development of personalized therapy and precision medical treatments.
\end{abstract}

Key words: bladder cancer, immune infiltration subtypes, The Cancer Genome Atlas, gene expression, CIBERSORT algorithm, personalized therapy

\section{Introduction}

As one of the most common types of urological malignancies, bladder cancer (BLCA) remains a major global medical problem despite the availability of numerous new treatment options. Transitional cell (urothelial) carcinoma is responsible for $95 \%$ of BLCA cases [1]. It is reported that there are 549,000 new cases of BLCA and 200,000 BLCA-related deaths per year in the world [2].
BLCA is highly heterogeneous on the genetic, expression, and histological [3]. Accurate understanding of this heterogeneity can promote the molecular classification of BLCA and the management of personalized medicine. Numerous studies have reported the influence of the immune microenvironment on BLCA development and immunotherapy including intravesical bacillus Calmette-Guérin (BCG) and PD-1/PD-L1 blockade 
was long applied for the treatment of BLCA $[4,5]$. The tumor microenvironment consists of immune cells, mesenchymal cells, endothelial cells, extracellular matrix (ECM) molecules, and inflammatory mediators [6]. BLCA is an immunosensitive tumor which is infiltrated by tumor-infiltrating immune cells (TIICs) including $\mathrm{T}$ cells, macrophages, dendritic cells, neutrophils and mast cells [7-9]. Studies have shown that the tumor microenvironment affects the gene expression of tumor tissues and the patient outcome, and therefore, has a diagnostic and prognostic value for BLCA [10-12]. TIICs, which are main components of tumor microenvironment, have been reported closely related to the effectiveness of targeted drugs and clinical outcomes. However, most studies evaluated TIICs based on immunohistochemical analysis, which relies on a single marker to identify a specific immune cell [11-14]. These traditional methods can be misleading and are not accurate as many marker proteins are not specific for different immune cells.

CIBERSORT is an algorithm to estimate specific cell types in a mixed cell population using gene expression data [15]. In the present study, gene expression data was obtained from The Cancer Genome Atlas (TCGA) bladder urothelial cancer dataset and the fractions of $22 \mathrm{immune}$ cell types were estimated by CIBERSORT. Four immune cell clusters with different clinical prognoses and mutation characteristics were identified by using unsupervised consensus clustering. It is hoped that this study may offer some important information for the understanding of the relationship between the heterogeneity of TIICs, and disease progression in BLCA, and provide insights into potential personalized therapeutic strategies for each subtype of BLCA.

\section{Materials and methods}

\section{Database and genomic analysis}

The mutation data, gene expression profiles, and clinical data of patients with BLCA were obtained from TCGA data portal (https://tcga-data.nci.nih. gov/tcga/). Gene expression data analysis was performed using the limma package of the R software. A fold change of $>2$ and false discovery rate (FDR) of $<0.05$ were used as cutoffs to identify differentially expressed genes (DEGs). The Maftools package was used to analyze and summarize the mutation data. Volcano plots and heat maps were generated using the ggplot2 and pheatmap packages, respectively.

\section{Evaluation of tumor-infiltrating immune cells}

CIBERSORT algorithm was used to calculate the fractions of infiltrating immune cells. CIBERSORT is an analytical tool that estimates specific cell types in a mixed cell population using gene expression data; the algorithm was run using the 1000 permutations and LM22 signature [16]. The fractions of immune cell were considered accurate when the CIBERSORT output reached $\mathrm{p}<0.05$.

\section{Characteristic genes enrichment analysis}

The clusterProfiler package was used to perform GO enrichment analysis [17]. An FDR of $<0.05$ was used as the cut-off value.

\section{Statistical analysis}

The unpaired $t$ test was used to assess the difference between the immune fractions from tumor and non-tumor tissues. The median of the proportion of each cell type was computed for survival analysis, and survival curves were constructed by the KaplanMeier method and compared using the means obtained from the log rank test. To investigate whether distinct classes of tumor-infiltrating immune cells are present in different tumors, the patients were clustered into four groups based on the consensus proportion of each cell type. The differences of tumor mutation burden (TMB) in case of genes from each cluster were analyzed by one-way ANOVA, followed by Tukey's multiple-comparison post-hoc test. All analyses were performed using the $\mathrm{R}$ software (version 3.6).

\section{Results}

\section{Composition and prognostic value of immune cells in bladder cancer}

Using the CIBERSORT algorithm, 22 subpopulations of immune cells in 199 samples (192 tumor tissues, 7 normal tissues) were investigated (Figure 1A). The fractions of M0 and M1 macrophages were higher in the tumor tissues than in the normal tissues, whereas the fractions of naive $B$ cells and resting mast cells were significantly lower in the tumor tissues (Figure 1B). M2 macrophages (13.7\%) were the most abundant infiltrating immune cells in BLCA, followed by M0 macrophages (13.2\%), CD8 T cells $(12.4 \%)$, and CD4 $\mathrm{T}$ cells $(10.2 \%)$. Then, Kaplan-Meier analysis and log-rank test were performed to analyze the prognostic value of the tumor-infiltrating immune cells. We found that CD8 T cells were associated with good prognosis (HR 0.571, 95\% CI 0.365-0.8932, p = 0.0149), whereas memory B cells (HR 1.765, 95\% CI 0.9926-3.138, p = 0.0221) were associated with poor prognosis in patients with BLCA (Figure 1C, D). 

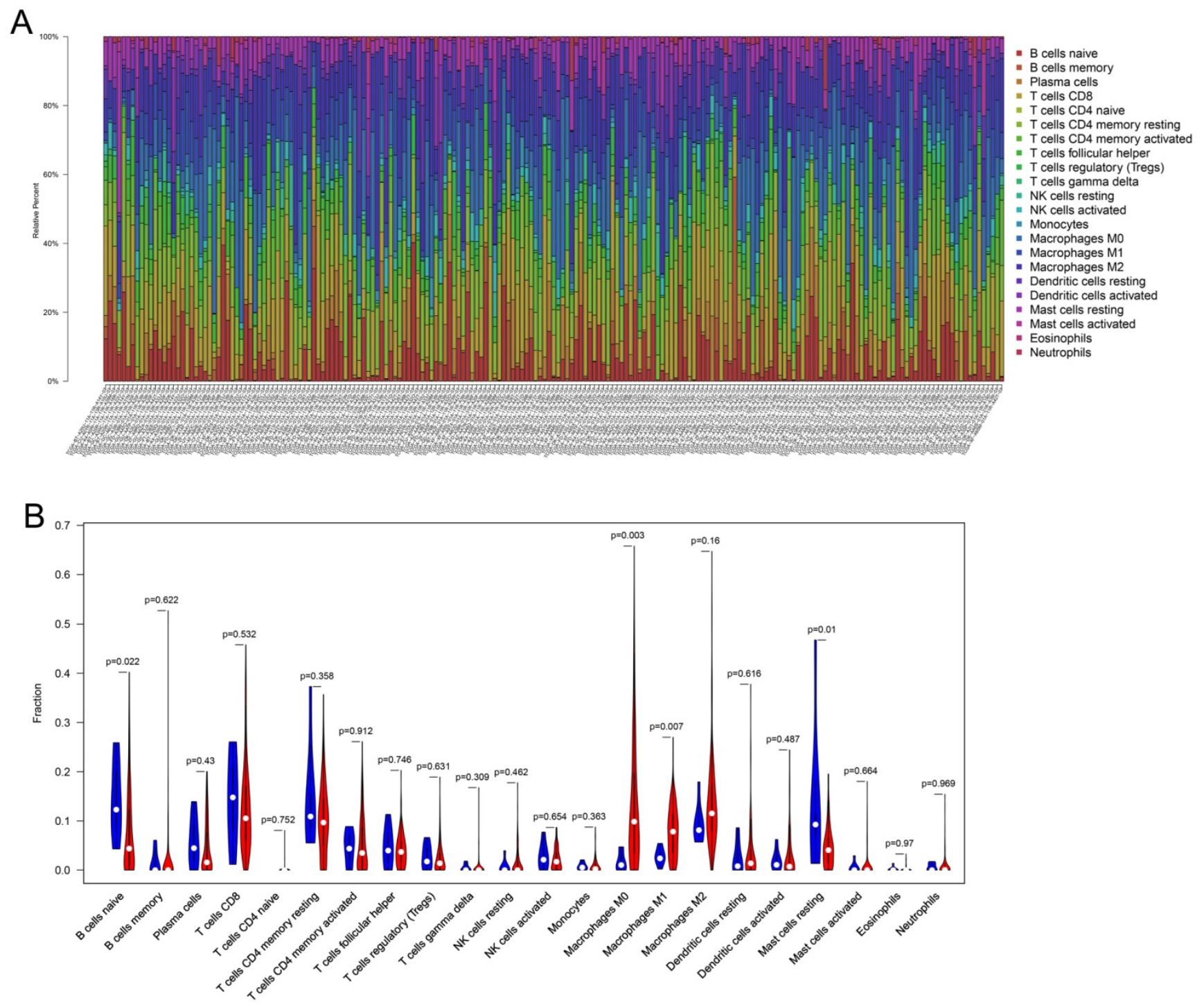

C

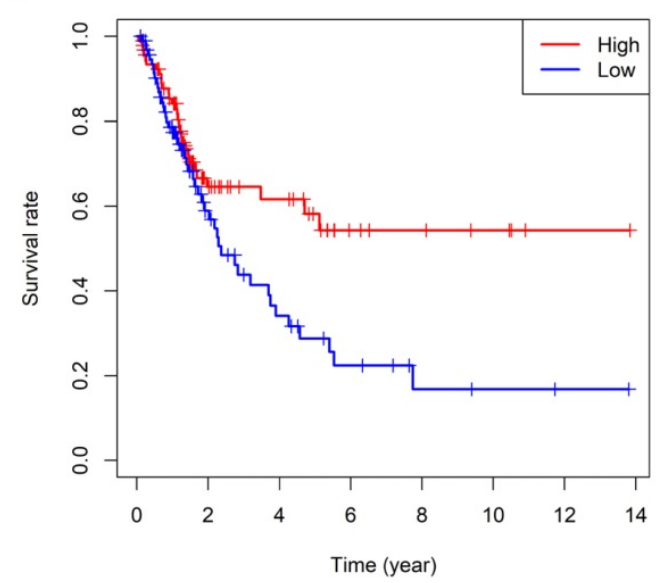

D

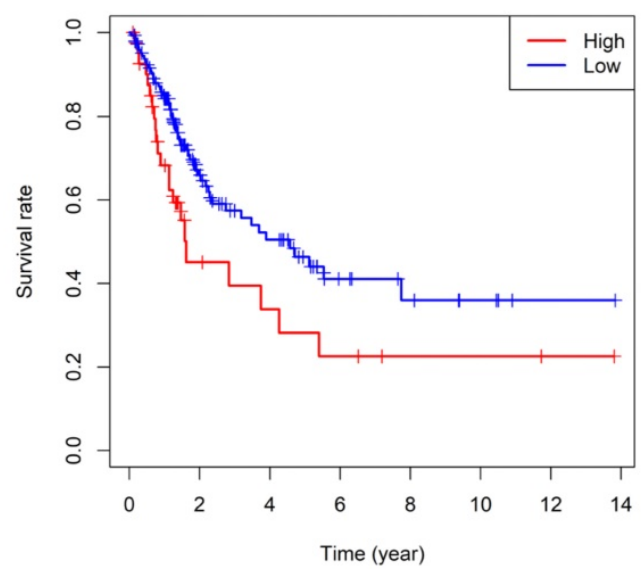

Figure 1. Composition and prognostic value of immune cells in bladder cancer (A) The percent of 22 types of fractions of tumor-infiltrating immune cell in bladder cancer. (B) 22 types of adaptive and innate immune cells in tumor and normal tissue groups. The fractions of M0 and M1 macrophages were consistently higher in the bladder cancer tissue than those of the normal tissue, whereas the fraction of naive $B$ cells and resting mast cells was significantly lower in bladder cancer tissue (by unpaired $t$ test). (C, D) The Kaplan-Meier survival curve of CD8 T cell and memory B cells in bladder cancer. Patients with high CD8 T cell fraction had a higher overall survival (HR $0.571,95 \% \mathrm{Cl}$ $0.365-0.8932, p=0.0149)$ whereas memory B cells $(H R 1.765,95 \% \mathrm{Cl} 0.9926-3.138, p=0.0221)$ were associated with poor prognosis. 


\section{Consensus clustering of immune cells identified four clusters of immune cell subtypes of BLCA}

The immune infiltration varies considerably at the individual level, and partially reflects the prognosis. Using unsupervised consensus clustering, we identified immune cell subtypes of BLCA with different clinical characters based on 22 different types of immune cells. Based on the similarity of immune infiltration, $\mathrm{k}=4$ was determined as the optimal number of clusters (Figure 2A, B). The consensus matrix heatmap revealed the four clusters that were identified (Figure 2C). The four clusters comprised different proportions of immune cells (Figure 2D). The fractions of the 22 immune cell types in each cluster are shown in Tables 1-4. Moreover, each cluster was associated with different clinical outcomes (Figure 2E). Significantly longer overall survival (OS) was found in the cluster 2 subgroup than that in other clusters.

Table 1. The fractions of tumor-infiltrating immune cells in cluster 1

\begin{tabular}{ll}
\hline Immune cells in cluster 1 & Fraction \\
\hline Macrophages M2 & 0.199609146 \\
T cells CD4 memory resting & 0.162765006 \\
Macrophages M0 & 0.093887564 \\
Macrophages M1 & 0.073074785 \\
T cells CD8 & 0.071416282 \\
Dendritic cells resting & 0.060103665 \\
Mast cells resting & 0.05985775 \\
B cells naive & 0.038816143 \\
Dendritic cells activated & 0.037608841 \\
T cells follicular helper & 0.029186935 \\
T cells regulatory (Tregs) & 0.028161336 \\
T cells CD4 memory activated & 0.02725125 \\
NK cells activated & 0.025787568 \\
Plasma cells & 0.020464493 \\
Mast cells activated & 0.01548266 \\
Monocytes & 0.015375007 \\
NK cells resting & 0.014180805 \\
Neutrophils & 0.013552612 \\
B cells memory & 0.009292636 \\
Eosinophils & 0.002728938 \\
T cells gamma delta & 0.001396577 \\
T cells CD4 naive & 0 \\
\hline
\end{tabular}

\section{Identification of the expression profile features of genes from the four clusters}

Since different clusters showed variations in the infiltrating immune cell types and patient outcomes, we explored the DEGs in the different clusters, compared to the genes in the normal tissues. In cluster 1, a total of 2694 DEGs (1689 upregulated and 1005 downregulated genes) were identified (Figure 3A) and visualized using a heatmap (Figure 3B). In cluster 2, a total of 3819 DEGs (2387 upregulated and 1432 downregulated genes) were identified (Figure 3C) and visualized using a heatmap (Figure 3D). In cluster
3, a total of 3260 DEGs (1984 upregulated and 1276 downregulated genes) were identified (Figure 3E) and visualized using a heatmap (Figure 3F). In cluster 4, a total of 3202 DEGs (2063 upregulated and 1139 downregulated genes) were identified (Figure 3G) and visualized using a heatmap (Figure $3 \mathrm{H}$ ).

Table 2. The fractions of tumor-infiltrating immune cells in cluster 2

\begin{tabular}{ll}
\hline Immune cells in cluster 2 & Fraction \\
\hline T cells CD8 & 0.233496 \\
Macrophages M2 & 0.120392 \\
T cells CD4 memory activated & 0.112792 \\
Macrophages M1 & 0.096799 \\
T cells CD4 memory resting & 0.066103 \\
Macrophages M0 & 0.05563 \\
T cells follicular helper & 0.054722 \\
Plasma cells & 0.041017 \\
Mast cells resting & 0.035316 \\
B cells naive & 0.03482 \\
Dendritic cells activated & 0.027455 \\
Dendritic cells resting & 0.027433 \\
NK cells resting & 0.025294 \\
T cells regulatory (Tregs) & 0.020309 \\
NK cells activated & 0.019001 \\
Monocytes & 0.010053 \\
B cells memory & 0.005778 \\
Neutrophils & 0.005571 \\
T cells gamma delta & 0.004365 \\
Mast cells activated & 0.002259 \\
Eosinophils & 0.001397 \\
T cells CD4 naive & 0 \\
\hline
\end{tabular}

\section{Identification of characteristic genes in the four clusters and functional enrichment analysis}

A total of 953 DEGs were found to be overlapping in the four clusters. A total of 120,461, 223 , and 517 characteristic genes were identified in cluster 1 , cluster 2 , cluster 3 , and cluster 4 , respectively (Figure 4A). GO enrichment analysis of the characteristic genes in cluster 1 indicated that these genes were involved in "nucleosome assembly", "chromatin assembly", and "nucleosome organization" in the 'biological process' category. "Nucleosome" was the most enriched term in the 'cellular components' category, while "protein heterodimerization activity" was the most significantly enriched term in the 'molecular function' category (Figure 4B). In case of the genes from cluster 2 , "antigen processing and presentation", "response to interferon-gamma", and "antigen processing and presentation of peptide antigen" were enriched in the 'biological process' category, "side of membrane", "coated vesicle membrane", and "MHC protein complex" were enriched in the 'cellular components' category, and "peptide binding" and "antigen binding" were enriched in the 'molecular function' category (Figure 4C). In case of genes from cluster 3, "keratinocyte differentiation" and "epidermal cell 
differentiation" were enriched in the "biological process' category, "cornified envelope" was enriched in the 'cellular components' category, and "cell adhesion molecule binding" was enriched in the 'molecular function' category (Figure 4D). In case of genes from cluster 4, "humoral immune response", "acute inflammatory response", and "regulation of

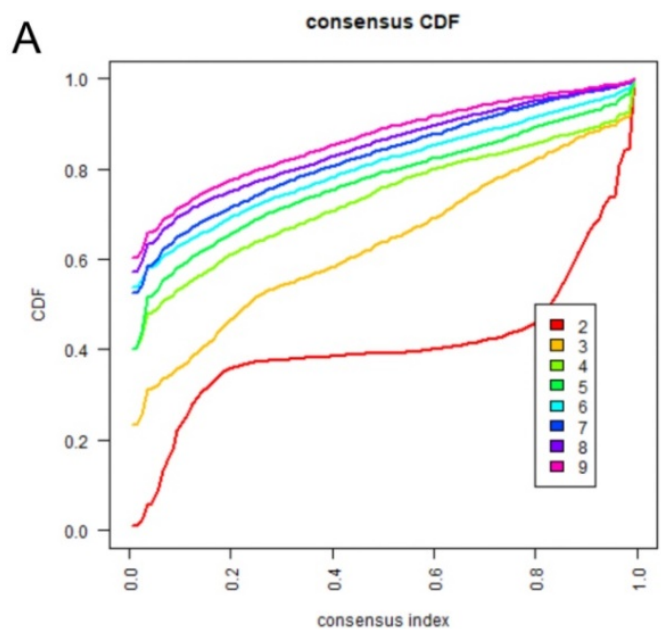

C
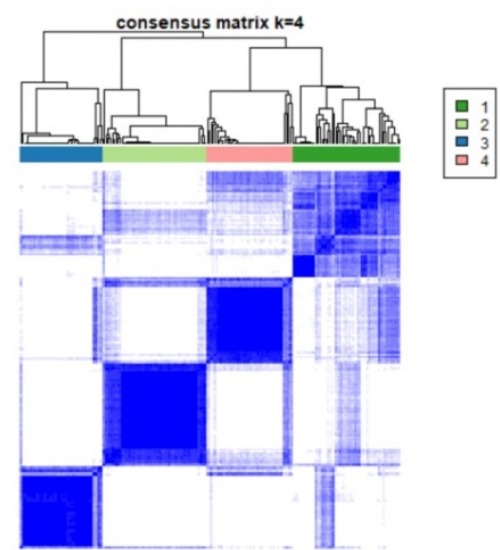

D

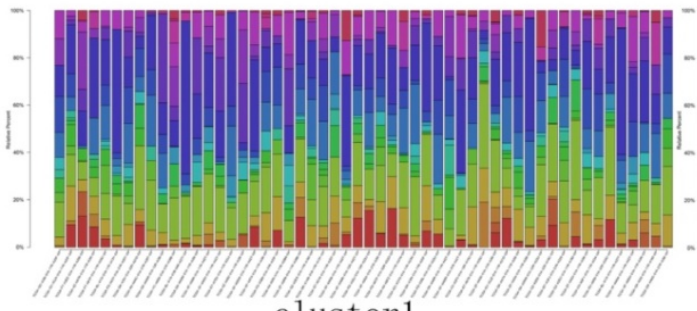

cluster1

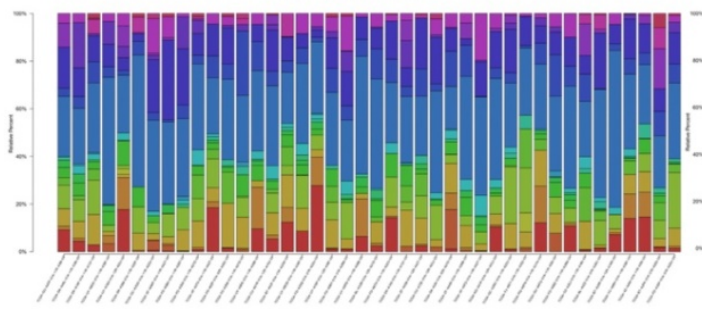

cluster 3 humoral immune response" were enriched in the 'biological process' category, no term was enriched in the 'cellular components' category, and "antigen binding and catalytic activity" and "acting on a glycoprotein" were enriched in the "molecular function' category (Figure 4E).

B

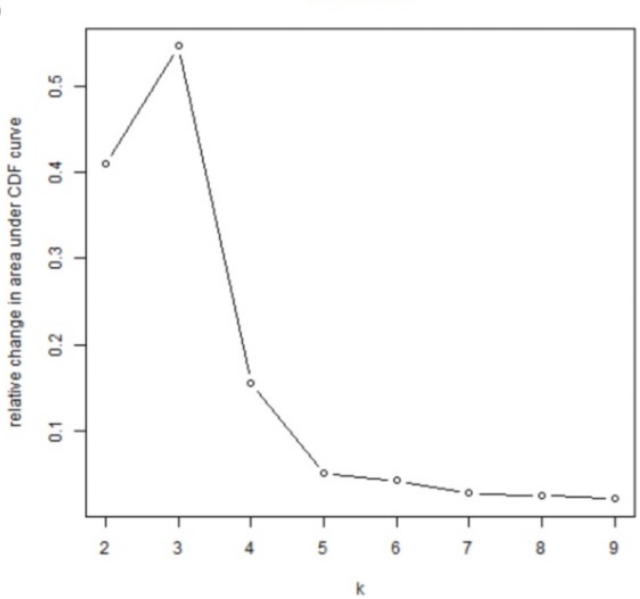

E

Survival curve $(p=0.047)$

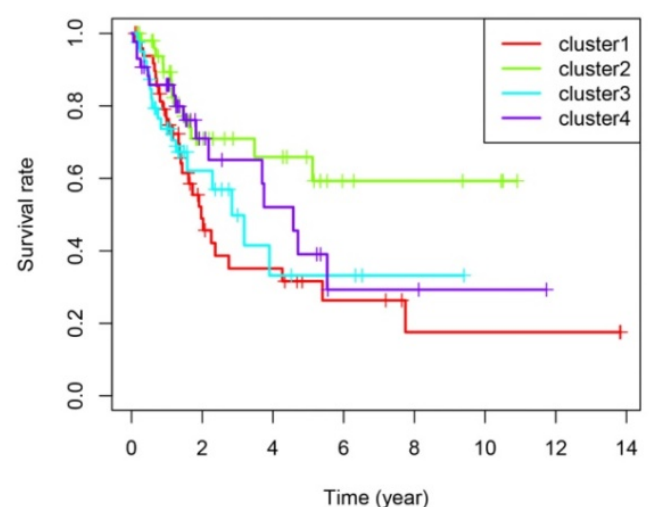

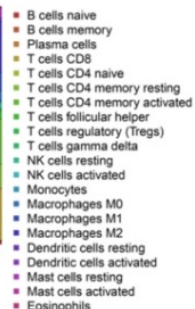

cluster 2

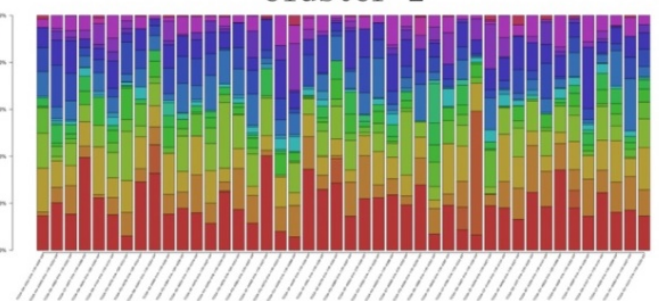

cluster 4

Figure 2. Consensus clustering of immune cells identified four clusters of bladder cancer. (A) Consensus clustering cumulative distribution function (CDF) for $k=$ 2 to 9 . (B) Relative change in area under CDF curve for $k=2$ to 9 . (C) Results of unsupervised consensus clustering identified four clusters. (D) The tumor-infiltrating immune cell proportions in four clusters. (E) The Kaplan-Meier survival curve of patients in different clusters. 

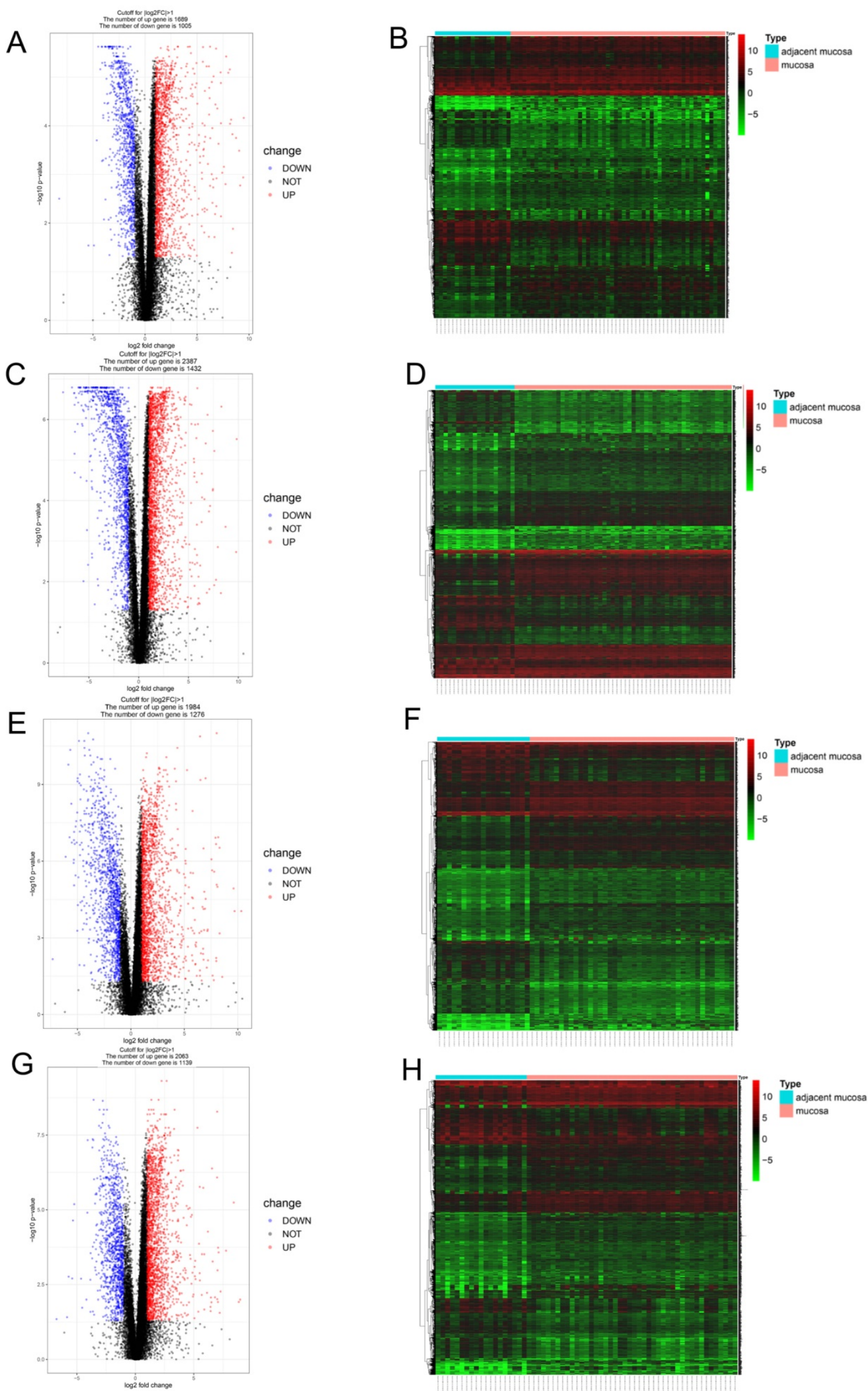

Figure 3. Identification of gene expression profile feature in four clusters. (A, B) The volcano plot and heatmap show the 2694 genes (1689 up-regulated and 1005 down-regulated) identified in cluster 1. (C, D) The volcano plot and heatmap show the 3819 genes (2387up-regulated and 1432 down-regulated) identified in cluster 2. (E, F) The volcano plot and heatmap show the 3260 genes (1984 up-regulated and 1276 down-regulated) identified in cluster 3 . (G, H) The volcano plot and heatmap show the 3202 genes (2063 up-regulated and 1139 down-regulated) identified in cluster 4. 
Table 3. The fractions of tumor-infiltrating immune cells in cluster 3

\begin{tabular}{ll}
\hline Immune cells in cluster 3 & Fraction \\
\hline Macrophages M0 & 0.335242 \\
Macrophages M2 & 0.124094 \\
T cells CD4 memory resting & 0.07798 \\
T cells CD8 & 0.074366 \\
Macrophages M1 & 0.070703 \\
B cells naive & 0.053255 \\
Mast cells resting & 0.040685 \\
T cells follicular helper & 0.040265 \\
Plasma cells & 0.030809 \\
T cells CD4 memory activated & 0.030375 \\
Dendritic cells resting & 0.024294 \\
NK cells activated & 0.021609 \\
Dendritic cells activated & 0.019461 \\
T cells regulatory (Tregs) & 0.011788 \\
Mast cells activated & 0.011171 \\
B cells memory & 0.010108 \\
NK cells resting & 0.009934 \\
Neutrophils & 0.004579 \\
T cells gamma delta & 0.003108 \\
T cells CD4 naive & 0.002469 \\
Monocytes & 0.002026 \\
Eosinophils & 0.00168 \\
\hline
\end{tabular}

Table 4. The fractions of tumor-infiltrating immune cells in cluster 4

\begin{tabular}{ll}
\hline Immune cells in cluster 4 & Fraction \\
\hline B cells naive & 0.194482 \\
T cells CD8 & 0.107602 \\
T cells CD4 memory resting & 0.093953 \\
Plasma cells & 0.09387 \\
Macrophages M2 & 0.093446 \\
Macrophages M0 & 0.074231 \\
Macrophages M1 & 0.067259 \\
Mast cells resting & 0.05007 \\
T cells follicular helper & 0.040493 \\
T cells CD4 memory activated & 0.035873 \\
T cells regulatory (Tregs) & 0.0339 \\
Dendritic cells activated & 0.032261 \\
B cells memory & 0.021131 \\
NK cells activated & 0.020878 \\
Dendritic cells resting & 0.01669 \\
Monocytes & 0.00695 \\
T cells gamma delta & 0.005126 \\
Neutrophils & 0.004548 \\
NK cells resting & 0.003773 \\
Mast cells activated & 0.003042 \\
Eosinophils & 0.000312 \\
T cells CD4 naive & 0.00011
\end{tabular}

\section{Identification of the mutation profile features of genes from each cluster}

It is well-known that cancer may results from the accumulation of somatic DNA mutations. High TMB leads to the formation of more new antigens, making tumors more immunogenic and more sensitive to immunotherapy. We downloaded the mutation data for each cluster from the TCGA database and calculated the respective TMB values. The results show that compared with the other clusters, cluster 2 had a higher TMB (Figure 5A). The alteration landscapes of the four clusters are shown in Figure 5B-E. In cluster 1, 6 genes were mutated by $>20 \%$ :
TP53 (52\%), TTN (35\%), RB1(23\%), KDM6A (21\%), KMT2C (21\%), and KMT2D (21\%). In cluster 2, 15 genes were mutated by > 20\%: TTN (65\%), TP53 (46\%), PIK3CA (40\%), MUC16 (38\%), KMT2D (35\%), ARID1A (31\%), HMCN1 (31\%), KMT2A (31\%), SYNE1 (29\%), RB1 (27\%), AHNAK (25\%), ERBB2 (25\%), KDM6A (21\%), KMT2C (21\%), and LRP1B $(21 \%)$. In cluster 3, 9 genes were mutated by $>20 \%$ : TP53 (55\%), KMT2D (33\%), TTN (31\%), ARID1A (26\%), KDM6A (24\%), XIRP2 (24\%), ELF3 (21\%), KMT2C (21\%), and PIK3CA (21\%). In cluster 4, 5 genes were mutated by > 20\%: TTN (36\%), KMT2D (34\%), TP53 (34\%), ARID1A (32\%), and MUC16 (27\%). There were fewer mutant genes in cluster 1, while cluster 2 had the greatest number of mutated genes (Figure 5F).

\section{Discussion}

While the introduction of novel targeted drugs can increase treatment options of BLCA, these treatments are only effective in certain BLCA patients. The identification of subtypes of BLCA will help biological research and personalized treatment of BLCA. TCGA is an open-access database that uses a genome-wide approach to reveal the genetic characteristics of cancers. Many studies on cancers such as BLCA have screened diagnostic and prognostic biomarkers using TCGA [18-20]. Patient subgroups with different treatment response and prognosis can also be identified using TCGA.

In the present study, four immune clusters for BLCA were identified and these four clusters showed different patterns of infiltrating immune cell gene expression signatures and mutation characteristics. Among all clusters, cluster 1 was associated with the worst prognosis, and cluster 2, with the best outcome. Cluster 1 was characterized by the increased infiltration of M2 macrophages and CD4 memory T cells. M2 macrophages play a role in antiinflammatory processes, tissue repair and remodeling, the immune regulation process, parasite clearance, and the tumor promotion process [21]. The immunosuppressive factors released by M2 macrophages may support immune evasion in bladder cancer. Tissue resident memory $\mathrm{T}$ cells are a key factor in making tumors dormant; hence, it is essential to establish a cancer-immune system balance [22]. The feature of cluster 2 was the increased infiltration of CD8 T cells. Studies have shown that the accumulation of CD8 $\mathrm{T}$ lymphocytes in tumors often indicates good clinical outcomes [23]. Under hypoxic conditions, CD8 T cells can differentiate into lytic effector cells, increase the expression of interferon gamma (IFNץ), Fas ligand (FASL), granule B (GZMB), and inhibit tumor cell proliferation [24,25]. 
M0 macrophages were the most abundant infiltrating immune cells in case of cluster 3 , and naive B cells were the most abundant infiltrating immune cells in case of cluster 4 . Previous studies have shown that activated naive $B$ cells are required for the initiation of
$\mathrm{T}$ cell immune responses, owing to their costimulatory activity and ability to produce cytokines for the activation and expansion of effector and memory TH cell populations $[26,27]$.
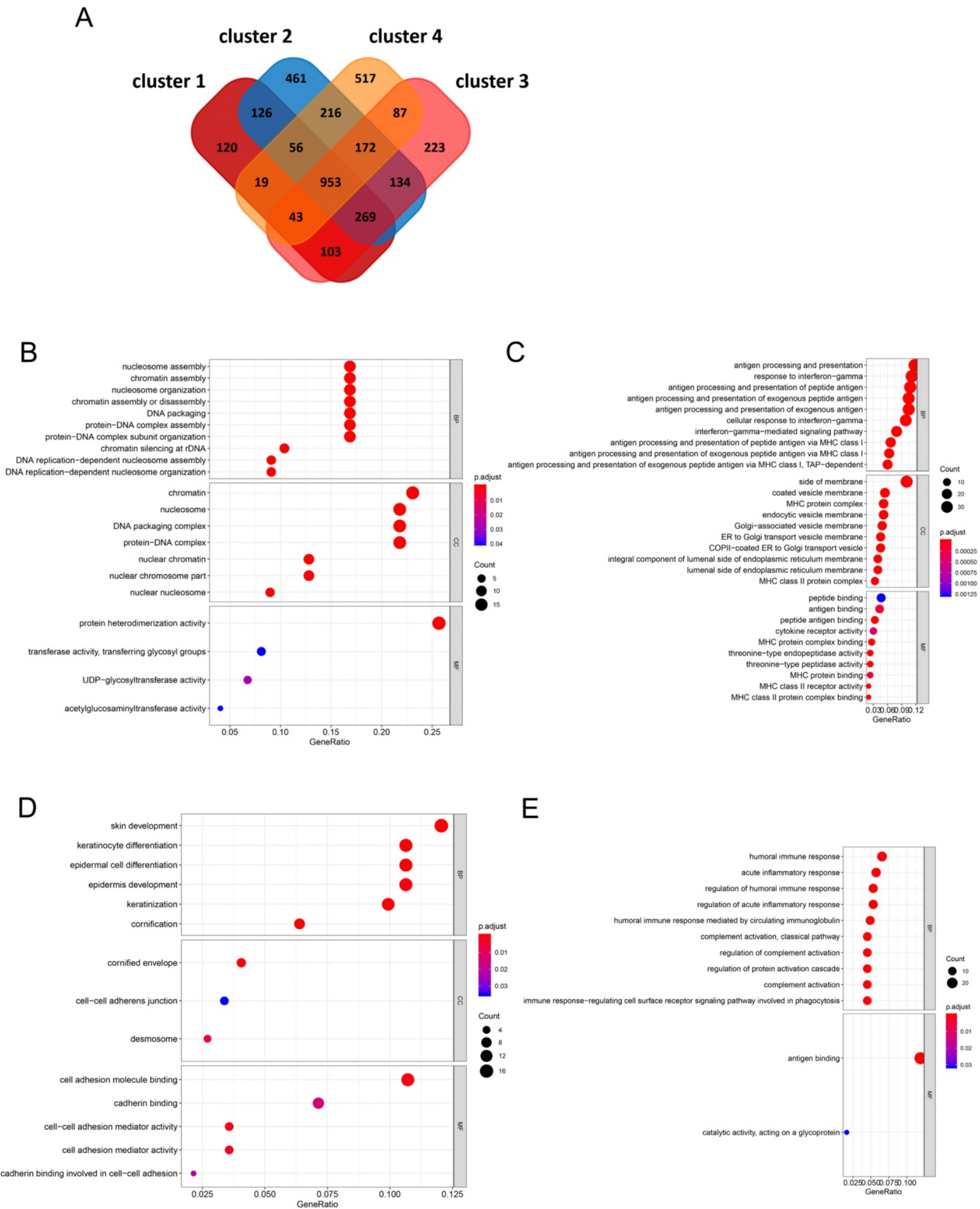

$E$

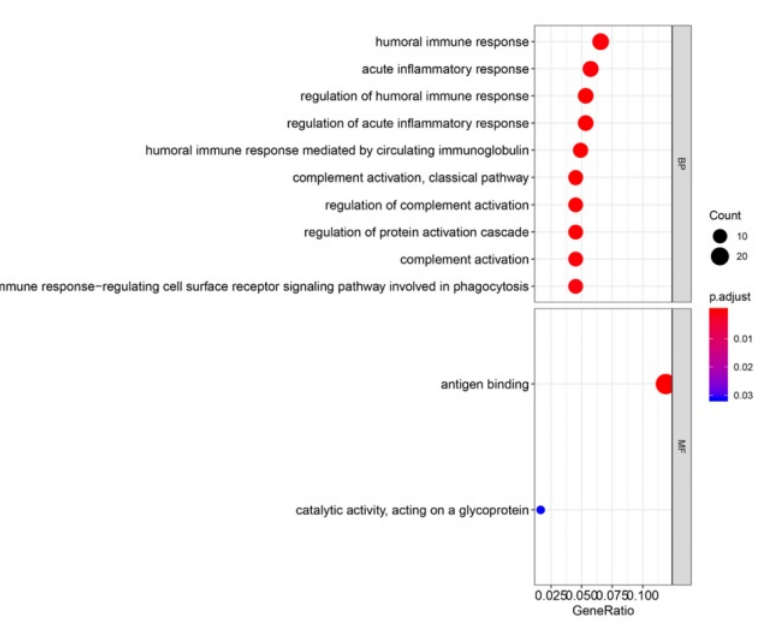

Figure 4. Identification of characteristic genes of four clusters and functional enrichment analysis (A) In the Venn diagrams, co-expression of upregulated and downregulated genes in four clusters. (B-E) The biological process, cellular component, and molecular function terms in four clusters. 
A

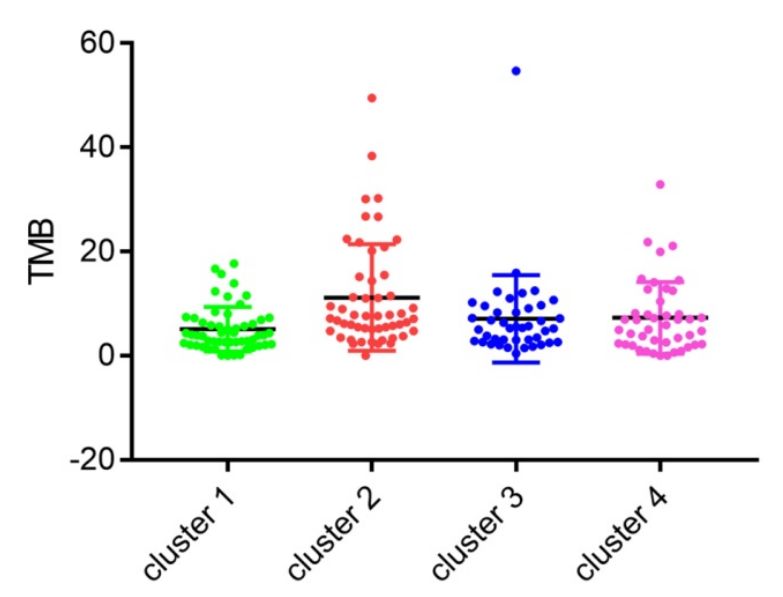

C

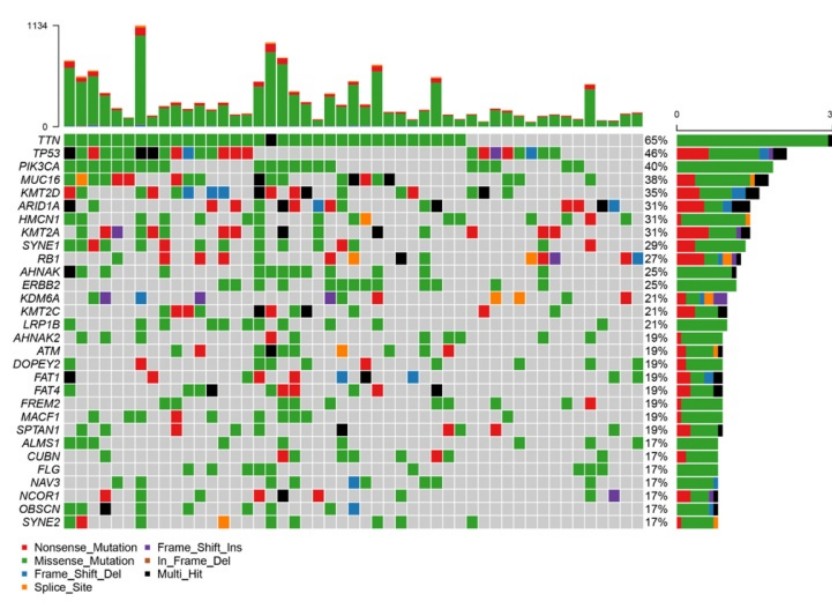

B

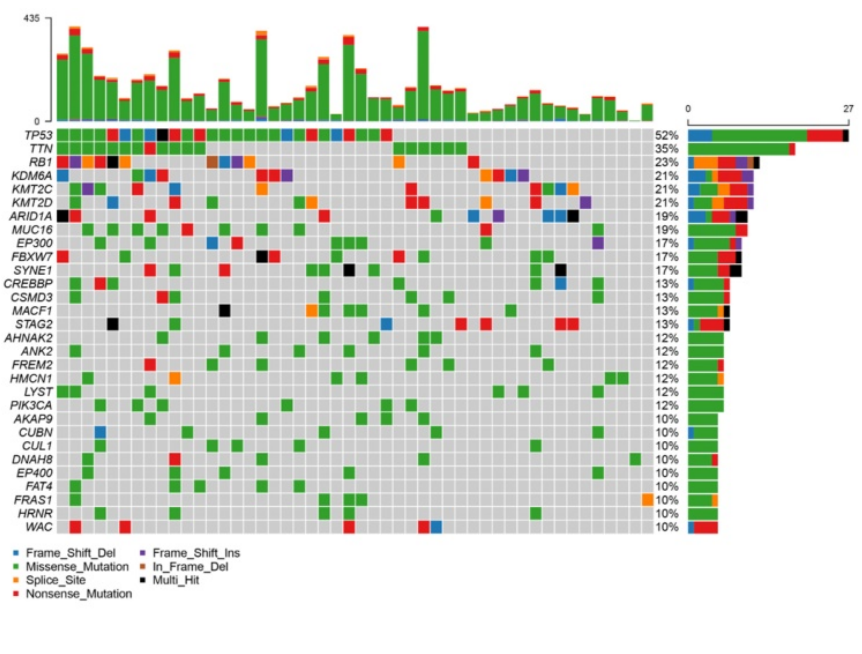

E

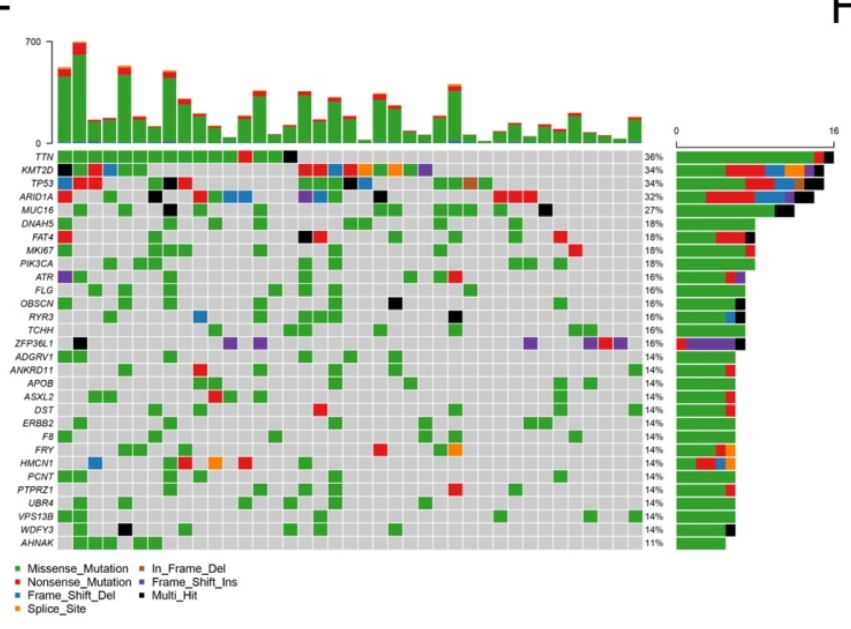

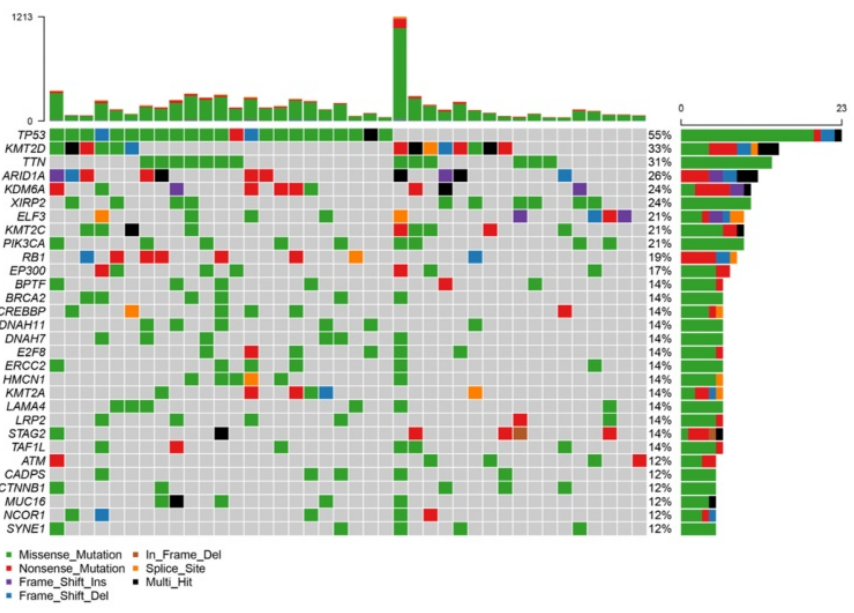

F
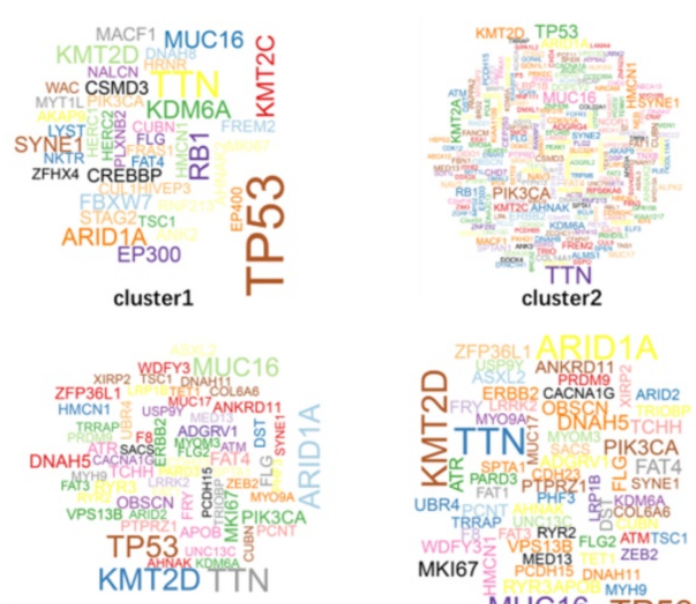

cluster3

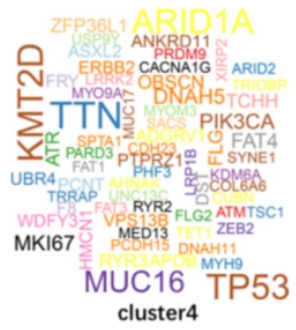

Figure 5. Identification of mutation profile feature of each cluster. (A) The TMB in four clusters. (B-E) The mutant genes and mutation profile of bladder cancer in four clusters. (F) Gene cloud map shows the name of mutant genes in four clusters. The size of gene names is proportional to the number of samples mutated for each gene.

The four subtypes also showed different gene expression signatures and were associated with different biological processes. The signature genes in cluster 1 were mainly involved in the process of cell division. The signature genes in cluster 2 were mainly involved in the response to interferon-gamma and 
antigen processing and presentation. The signature genes in cluster 3 were mainly involved in epidermal cell differentiation. The signature genes in cluster 4 were mainly involved in the humoral immune response. This result reflects a profound link between gene expression and immune cell infiltration. Cancer is a genetic disease and caused by the accumulation of somatic mutations [28]. In a sense, the characteristics of immune infiltration also represent the genetic characteristics of cancer. Next, we analyzed the mutation characteristics of genes from each cluster. The results showed that the mutant genes and mutation frequencies in each cluster were different. In cluster 1, 6 genes were mutated by $>20 \%$ : TP53 (52\%), TTN (35\%), RB1 (23\%), KDM6A (21\%), KMT2C (21\%), and KMT2D (21\%). In cluster 2, 15 genes were mutated by > 20\%: TTN (65\%), TP53 (46\%), PIK3CA (40\%), MUC16 (38\%), KMT2D (35\%), ARID1A (31\%), HMCN1 (31\%), KMT2A (31\%), SYNE1 (29\%), RB1 (27\%), AHNAK (25\%), ERBB2 (25\%), KDM6A (21\%), KMT2C (21\%), and LRP1B (21\%). In cluster 3, 9 genes were mutated by > 20\%: TP53 (55\%), KMT2D (33\%), TTN (31\%), ARID1A (26\%), KDM6A (24\%), XIRP2 (24\%), ELF3 (21\%), KMT2C (21\%), and PIK3CA (21\%). In cluster 4, 5 genes were mutated by $>20 \%$ : TTN (36\%), KMT2D (34\%), TP53 (34\%), ARID1A (32\%), and MUC16 (27\%).

We found that TP53 and TTN are the major mutant genes in cluster 1 . The tumor suppressor gene TP53 has been reported to be mutated in more than $50 \%$ of human malignancies, and thus, promote the development and progression of cancer [29]. In comparison with other groups cluster 2 have more mutated gene and a higher TMB. It has been observed in other tumors that high TMB may reflects the presence of new antigens, thereby increasing lymphocyte infiltration in the tumor microenvironment [30,31]. The cluster 3 had similar mutation characteristics with cluster 1; TP53 and TTN were the major mutant genes. Additionally, we found that the mutation in TP53 in case of cluster 4 is significantly lower than that in case of the other clusters. Furthermore, there was no dominant mutant gene in cluster 4 . TP53 mutations often result in unstable tumor genomes and impaired DNA repair capacity, therefore; TP53 mutant tumors may be more sensitive to DNA damage factors [3]. Conversely, a wild-type TP53 gene expression signature in BLCA have been shown to be resistant to neoadjuvant chemotherapy [32], so the prognosis of patients in this cluster 4 may likely to be resistant to neoadjuvant chemotherapy. BLCA is one of the most immunotherapy-responsive solid tumors. High TMB is associated with the response to immune checkpoint inhibitors (ICIs) in BLCA [33]. Patients in cluster 2 have high infiltration of CD8 T cells. Moreover, high levels of TMB suggest that these patients may benefit from ICIs, such as anti-PD-1 or anti-CTLA-4 therapies. Cluster 3 was highly expressed basal and squamous differentiation markers. Basal bladder cancer originates from basal cells and stem cells of the bladder urothelium [34], which characterized by enrichment of squamous, stemness and EMT markers [35]. This subtype may be more sensitive to neoadjuvant chemotherapy. In basal BLCA, epidermal growth factor receptor (EGFR) pathway was usually activated [36]. Therefore, anti-EGFR therapy may provide benefits for patients of this subtype.

In conclusion, based on immune cell types, we used unsupervised consensus clustering to identify four subtypes of BLCA. These four immune subtypes showed distinct mRNA expression patterns and different mutation characteristics. These differences may affect the development of the tumors and the sensitivity towards the treatment. We also provided a potential treatment strategy for different subtypes. This study may be helpful for the exploration of personalized treatments.

\section{Acknowledgments}

This study was supported by the National Natural Science Foundation of China (Grant No. 81470987 and NO. 81900637), the Tai Shan Scholar Foundation to Benkang Shi and Primary Research \& Development Plan of Shandong Province (2017GSF221010 to Y. Zhu).

\section{Competing Interests}

The authors have declared that no competing interest exists.

\section{References}

1. $\mathrm{Wu}$ XR. Urothelial tumorigenesis: a tale of divergent pathways. Nat Rev Cancer. 2005;5(9):713-725.

2. Bray F, Ferlay J, Soerjomataram I, Siegel RL, Torre LA, Jemal A. Global cancer statistics 2018: GLOBOCAN estimates of incidence and mortality worldwide for 36 cancers in 185 countries. CA Cancer J Clin. 2018;68(6):394-424.

3. Alifrangis C, McGovern U, Freeman A, Powles T, Linch M. Molecular and histopathology directed therapy for advanced bladder cancer. Nat Rev Urol. 2019;16(8):465-483

4. Eich ML, Chaux A, Guner G, et al. Tumor immune microenvironment in non-muscle invasive urothelial carcinoma of bladder. Hum Pathol. 2019.

5. Qiu S, Deng L, Liao X, et al. Tumor-associated macrophages promote bladder tumor growth through PI3K/AKT signal induced by collagen. Cancer Sci. 2019.

6. Hanahan D, Coussens LM. Accessories to the crime: functions of cells recruited to the tumor microenvironment. Cancer Cell. 2012;21(3):309-322.

7. Schwamborn K, Ammann JU, Knüchel R, et al. Multicentric analytical comparability study of programmed death-ligand 1 expression on tumor-infiltrating immune cells and tumor cells in urothelial bladder cancer using four clinically developed immunohistochemistry assays. Virchows Arch. 2019.

8. Stone L. Bladder cancer: Mastering the immune microenvironment. Nat Rev Urol. 2017;14(11):639.

9. Sweis RF, Spranger S, Bao R, et al. Molecular Drivers of the Non-T-cell-Inflamed Tumor Microenvironment in Urothelial Bladder Cancer. Cancer Immunol Res. 2016;4(7):563-568. 
10. Efstathiou JA, Mouw KW, Gibb EA, et al. Impact of Immune and Stromal Infiltration on Outcomes Following Bladder-Sparing Trimodality Therapy for Muscle-Invasive Bladder Cancer. Eur Urol. 2019;76(1):59-68.

11. Liu $\mathrm{Z}, \mathrm{Zhu} \mathrm{Y}, \mathrm{Xu} \mathrm{L}$, et al. Tumor stroma-infiltrating mast cells predict prognosis and adjuvant chemotherapeutic benefits in patients with muscle invasive bladder cancer. Oncoimmunology. 2018;7(9):e1474317.

12. Xue $Y$, Tong L, LiuAnwei LF, et al. Tumor-infiltrating M2 macrophages driven by specific genomic alterations are associated with prognosis in bladder cancer. Oncol Rep. 2019;42(2):581-594.

13. Ayari $\mathrm{C}$, LaRue $\mathrm{H}$, Hovington $\mathrm{H}$, et al. High level of mature tumor-infiltrating dendritic cells predicts progression to muscle invasion in bladder cancer. Hum Pathol. 2013;44(8):1630-1637.

14. Zhou L, Xu L, Chen L, et al. Tumor-infiltrating neutrophils predict benefit from adjuvant chemotherapy in patients with muscle invasive bladder cancer. Oncoimmunology. 2017;6(4):e1293211.

15. Chen B, Khodadoust MS, Liu CL, Newman AM, Alizadeh AA. Profiling Tumor Infiltrating Immune Cells with CIBERSORT. Methods Mol Biol. 2018;1711:243-259.

16. Newman AM, Liu CL, Green MR, et al. Robust enumeration of cell subsets from tissue expression profiles. Nat Methods. 2015;12(5):453-457.

17. Yu G, Wang LG, Han Y, He QY. clusterProfiler: an R package for comparing biological themes among gene clusters. OMICS. 2012;16(5):284-287.

18. Kuang S, Li H, Feng J, Xu S, Le Y. Correlation of BRCA2 gene mutation and prognosis as well as variant genes in invasive urothelial carcinoma of the bladder. Cancer Biomark. 2019;25(2):203-212.

19. Morera DS, Lahorewala SS, Belew D, et al. Clinical parameters outperform molecular subtypes for predicting outcome in bladder cancer: Results from multiple cohorts including TCGA. J Urol. 2019:101097JU0000000000000351.

20. Wang SS, Chen G, $\mathrm{Li} \mathrm{SH}$, et al. Identification and validation of an individualized autophagy-clinical prognostic index in bladder cancer patients. Onco Targets Ther. 2019;12:3695-3712.

21. Cheng H, Wang Z, Fu L, Xu T. Macrophage Polarization in the Development and Progression of Ovarian Cancers: An Overview. Front Oncol. 2019;9:421.

22. Gabriel SS, Kallies A. Tissue-resident memory $\mathrm{T}$ cells keep cancer dormant. Cell Res. 2019;29(5):341-342.

23. Fridman WH, Pagès F, Sautès-Fridman C, Galon J. The immune contexture in human tumours: impact on clinical outcome. Nat Rev Cancer. 2012:12(4):298-306.

24. de Silly R V, Ducimetière L, Yacoub MC, Dietrich PY, Derouazi M, Walker PR. Phenotypic switch of CD8(+) T cells reactivated under hypoxia toward IL-10 secreting, poorly proliferative effector cells. Eur J Immunol. 2015;45(8):2263-2275

25. Xu Y, Chaudhury A, Zhang M, et al. Glycolysis determines dichotomous regulation of T cell subsets in hypoxia. J Clin Invest. 2016;126(7):2678-2688.

26. LeBien TW, Tedder TF. B lymphocytes: how they develop and function. Blood. 2008;112(5):1570-1580.

27. Wu XZ, Shi XY, Zhai K, et al. Activated naïve B cells promote development of malignant pleural effusion by differential regulation of TH1 and TH17 response. Am J Physiol Lung Cell Mol Physiol. 2018;315(3):L443-443L455.

28. Chan TA, Yarchoan M, Jaffee E, et al. Development of tumor mutation burden as an immunotherapy biomarker: utility for the oncology clinic. Ann Oncol. 2019;30(1):44-56

29. VJN B, Eriksson SE, Bianchi J, Wiman KG. Targeting mutant p53 for efficient cancer therapy. Nat Rev Cancer. 2018;18(2):89-102.

30. Innocenti $\mathrm{F}, \mathrm{Ou} \mathrm{FS}, \mathrm{Ou} \mathrm{X}$, et al. Mutational Analysis of Patients With Colorectal Cancer in CALGB/SWOG 80405 Identifies New Roles of Microsatellite Instability and Tumor Mutational Burden for Patient Outcome. J Clin Oncol. 2019;37(14):1217-1227.

31. Wang X, Li M. Correlate tumor mutation burden with immune signatures in human cancers. BMC Immunol. 2019;20(1):4.

32. Choi W, Porten S, Kim S, et al. Identification of distinct basal and luminal subtypes of muscle-invasive bladder cancer with different sensitivities to frontline chemotherapy. Cancer Cell. 2014;25(2):152-165.

33. Mariathasan S, Turley SJ, Nickles D, et al. TGF $\beta$ attenuates tumour response to PD-L1 blockade by contributing to exclusion of $\mathrm{T}$ cells. Nature. 2018;554(7693):544-548.

34. Choi W, Ochoa A, McConkey DJ, et al. Genetic Alterations in the Molecular Subtypes of Bladder Cancer: Illustration in the Cancer Genome Atlas Dataset. Eur Urol. 2017;72(3):354-365.

35. Aine M, Eriksson P, Liedberg F, Sjödahl G, Höglund M. Biological determinants of bladder cancer gene expression subtypes. Sci Rep. 2015;5:10957.

36. Rebouissou S, Bernard-Pierrot I, de Reyniès A, et al. EGFR as a potential therapeutic target for a subset of muscle-invasive bladder cancers presenting a basal-like phenotype. Sci Transl Med. 2014;6(244):244ra91. 